\title{
Epidemiological data and antifungal susceptibility in invasive fungal infections - a Romanian infectious diseases tertiary hospital's experience. Preliminary report
}

\author{
Radu Agrosoaie ${ }^{1 *}$, Adrian Streinu-Cercel ${ }^{2,3}$, Doina Azoicai ${ }^{1}$, Codrina Bejan $^{1}$, \\ Olga Dorobat ${ }^{3}$, Alexandru Mihai ${ }^{3}$, Mona Popoiu ${ }^{3}$, Alexandru Rafila ${ }^{2,3}$ \\ 1. "Grigore T. Popa” University of Pharmacy and Medicine Iasi \\ 2. "Carol Davila” University of Medicine and Pharmacy Bucharest \\ 3. "Prof. Dr. Matei Bals” National Institute of Infectious Diseases Bucharest
}

\begin{abstract}
Introduction: Invasive fungal infections have stood as an important research subject for the past 20 years, being considered as a crucial effect of advancing healthcare services. Low identification rates of invasive fungal infections in blood cultures and low sensibility of biomarkers determine empiric treatments which lead to a change in epidemiological data and antifungal susceptibility.

The aim: The epidemiological evaluation of invasive fungal infections and the assessment of antifungal resistance related to this condition.

Methods and material: An "antifungal stewardship" retrospective study was developed between January 2010 and April 2016. An epidemiological analysis was performed on 79 cases with proven invasive fungal infections in bloodstream, catheter, and cerebrospinal fluid. We considered: age, gender, HIV status, place of residence, and first option in medical practice of antifungal treatment. The laboratory analysis was performed by the Microbiology Laboratory at "Prof. Dr. Matei Bals" National Institute for Infectious Diseases, Bucharest. Minimum inhibitory concentrations (MIC's) of 15 isolates were identified using colorimetric micro broth dilution panel YEASTONE ${ } Y O 10$ and compared with susceptibilities obtained by VITEK2 ${ }^{\circledR} C$ system. Candida parapsilosis ATCC 22019 was used as reference.

Results: The incidence of invasive fungal infections was 3.7 on 1000 hospitalized patients. The age of the study population ranged between 12 and 83 years, and most were male (59\%). The majority of subjects were from an urban area (84\%), and $27 \%$ of them were HIV positive. The results obtained in VITEK2C ${ }^{\circledR}$ were similar with those from YEASTONE® YO10 for fluconazole, voriconazole, amphotericin B (100\%), without any minor, major or very major errors. The fluconazole was the first option of treatment, followed by voriconazole, caspofungin, anidulafungin. In 37\% of cases the first treatment option was replaced with a secondary antifungal therapy accordingly

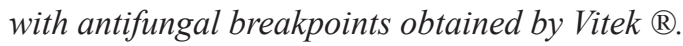

*Corresponding author: Radu Agrosoaie, "Grigore T. Popa” University of Pharmacy and Medicine Iasi, Romania. E-mail: agrosoaie@yahoo.com 
Conclusions: No rates of resistance to fluconazole, amphotericin B, voriconazole were obtained. Fluconazole was the major first line antifungal therapy.

Keywords: invasive fungal infections, antifungal stewardship, VITEK2 ${ }^{\circledR}, Y E A S T O N E ® Y O 10$

Received: $2^{\text {nd }}$ March 2017; Accepted: 12 ${ }^{\text {th }}$ June 2017; Published: $21^{\text {st }}$ August 2017

\section{Introduction}

Resistance to antibiotics and antifungals represents a bigger threat than the financial crisis in 2008. Predicted estimations announce that $10,000,000$ people can die due to this phenomenon with major impact for demography, economics (10 trillion USD), and healthcare [1]. There is a worldwide scientific dedication to analyze epidemiological data for each country, area, hospital, and department in order to control this phenomenon known as 'antifungal or antibiotic stewardship'. Especially in IFI, where the late and low identification rates of invasive fungal infections (IFI) in blood cultures, debatable specificity and sensibility of biomarkers and the high mortality of these infections, determined medical practitioners to administrate the empirical therapy along with prophylactic usage on patients at risk. This overuse of empirically and prophylactic treatments generate resistance and species distribution changes. The most common fungal infections of hospitalized patients are invasive candidiasis. Of all Candida species, 15 are involved in pathogenicity. Around $90 \%$ of invasive infections are determined by $C$. albicans, C. glabrata, C. tropicalis, C. parapsilosis, and $C$. krusei [2] with a shift from C.albicans in favor of non-albicans in recent years, due to high presence in intensive care patients predicted to be between 0.5 and $10 \%$ [3]. The lowest presence is in patients with neoplasm and transplant of hematopoietic stem cells from $0.15 \%$ to $1.55 \%$ [4]. Some antifungals do not have efficiency against some species, such as C.krusei to fluconazole. Globally, researchers are facing a risk of 33\% high resistance to fluconazole and also to echinocandins (for example anidulafungin, percentage of resistance is close to 6.4\%) [5]. C.glabrata has a high potential of resistance to echinocandins and was detected predominantly in the bloodstream of patients with infections associated with healthcare (HAI). Resistance was detected in a percentage of $5.1 \%$ for caspofungin, $3.8 \%$ to anidulafungin, $3.2 \%$ to micafungin, $7.7 \%$ for fluconazole, $5.1 \%$ for posaconazole, and $6.4 \%$ for voriconazole [6]. In Romania, clear evaluations regarding susceptibility are limited, excepting one multicenter study regarding susceptibility pattern of 551 species from bloodstream (BSI), superficial and deep-seated fungal infections which identified a resistance of $10.2 \%$ to fluconazole and $2.5 \%$ to voriconazole globally, and $4 \%$ in BSI [7]. This constituted the rationale to develop an analysis of antifungal susceptibilities in Romania for IFI. In order to identify susceptibility to antifungals we can use options such as automatic systems (VITEK $2 \mathrm{C}($ ) or visual identifications compared with a scale like YEASTONE®, E-TEST ${ }^{\circledR}$. Vitek $2 \mathrm{C} \circledast$ represents an automatic system of identification of germs and susceptibility offering the "breakpoints" associated with minimum inhibitory concentration (MIC). Studies showed that IFI with C. albicans having a value of MIC over $2 \mathrm{mg} / \mathrm{L}$ had a high rate of mortality. This means that it is important to see the level for our isolates in each center [8].

\section{Material and method}

In order to evaluate the susceptibility of antifungals and options for antifungal agents we retrospectively analyzed all the isolates on fungal bloodstream infections (BSI), catheter colonization and cerebrospinal fluid infections detected by VITEK 2 assessed at the microbiology laboratory in the National Institute for Infectious 
Diseases "Prof. Dr. Matei Bals "Bucharest, from January 2010 to April 2016. The isolates responsible for invasive fungal infections, grown in Bact/Allert systems were identified and tested to antifungals panel of VITEK $2 \AA$ correlated with EUCAST. Those isolates were stored in a strain collection bank. In 2016 those isolates were cultivated on Sabouraud agar and incubated 24 to 48 hours at $35^{\circ} \mathrm{C}$. Small colonies were passed subsequently on YEASTONE, a colorimetric technology which represents micro broth dilutions used to detect susceptibility to Candida, Aspergillus, Cryptococcus and other fungi with rapid growth (Instructions for Use Thermo Scientific SensititreYeastOne ${ }^{\circledR}$ (SYO) Susceptibility Plates, 2015) [9]. As reference strain C. parapsilosis ATCC 22019 was used. The option was for SYO, YO10 type due to its structure of antifungals present on the Romanian pharmaceutical market (excepting Amphotericin B and 5-Flucytozine). Plate inoculation was done accordingly to the manufacturer's instructions. The interpretation was done visually. We identified within the microbiology lab files the susceptibility to antifungals offered by VITEK2 $\AA$ and compared to YEASTONE $₫$ in order to track errors (minor, major, very major error).

\section{Results}

We evaluated 79 isolates identified in proven IFI and only 15 isolates were recovered after passing on Sabouraud medium from the 20102016 collection bank. The rate of incidence of IFI was evaluated at 3.7 on 1,000 hospitalized patients. Regarding epidemiological descriptive data, this study evaluated age, gender, HIV status. The age in this study (medium age) was 41 years and the extreme ages were 12 years old and 83 years old. Male gender was predominant with $59 \%$. Most of the patients came from from urban areas. The rate of HIV infection in this IFI study group was $27 \%$.
In the present study 15 isolates grown on $\mathrm{Sa}$ bouraud, transferred on YEASTONE® YO10MIC's were incubated at $35^{\circ} \mathrm{C}$ and the results were visually read after the legend provided by the producer. All the selected samples had a positive control marker. Regarding the accuracy of the method, no errors were noticed.

The comparison of the values of MIC's of the same isolates obtained on YEASTONE and MIC's obtained by automated system VITEK2C ${ }^{\circledR}$ (breakpoints) are presented in tables I and table II.

The range of MIC's on YEASTONE® YO10 varied as follows: fluconazole between $\leq 0.06 \mathrm{mcg} / \mathrm{ml}$ and $0.25 \mathrm{mcg} / \mathrm{ml}$, voriconazole $\leq 0.008 \mathrm{mcg} / \mathrm{ml}$ and $0.25 \mathrm{mcg} / \mathrm{ml}$, posaconazole $0.015 \mathrm{mcg} / \mathrm{ml}$ and $1 \mathrm{mcg} / \mathrm{ml}$, itraconazole $\leq 0.015 \mathrm{mcg} / \mathrm{ml}$ and $0.50 \mathrm{mcg} / \mathrm{ml}$, caspofungin between $0.015 \mathrm{mcg} / \mathrm{ml}$ and $0.50 \mathrm{mcg} / \mathrm{ml}$, anidulafungin $0.015 \mathrm{mg} / \mathrm{ml}$ and $2 \mathrm{mg} / \mathrm{ml}$, micafungin $\leq 0.008 \mathrm{mcg} / \mathrm{ml}$ and $2 \mathrm{mcg} / \mathrm{ml}$, amphotericin B between $\leq 0.12 \mathrm{mcg} / \mathrm{ml}$ and $0.5 \mathrm{mcg} / \mathrm{ml}, 5$-flucytozine $0.25 \mathrm{mcg} / \mathrm{ml}$ to $8 \mathrm{mcg} / \mathrm{ml}$.

The range of MIC's identified on VITEK2 ${ }^{\circledR}$ (breakpoints) varied as follows: fluconazole between $\leq 1 \mathrm{mcg} / \mathrm{ml}$ and $2 \mathrm{mcg} / \mathrm{ml}$, voriconazole $\leq 0.12 \mathrm{mcg} / \mathrm{ml}$, caspofungin between $\leq 0.25$ $\mathrm{mcg} / \mathrm{ml}$ and $1 \mathrm{mcg} / \mathrm{ml}$, micafungin $\leq 0.06 \mathrm{mcg} /$ $\mathrm{ml}$ and $0.50 \mathrm{mcg} / \mathrm{ml}$, amphotericin B between $\leq 0.25 \mathrm{mcg} / \mathrm{ml}$ and $1 \mathrm{mcg} / \mathrm{ml}$.

This present study compared the MIC from tables I and II generated by YEASTONE $®$ with EUCAST standards (8.0 version, November 2015) and versus CLSI standards (2012 version) and obtained -S - susceptible, I - intermediary, $\mathrm{R}$ - resistant.

The susceptibilities were compared in table III, table IV and table V with susceptibilities offered by VITEK 2C system. No errors were noticed.

The first options for antifungal treatment, proportionally, were represented by fluconazole, 


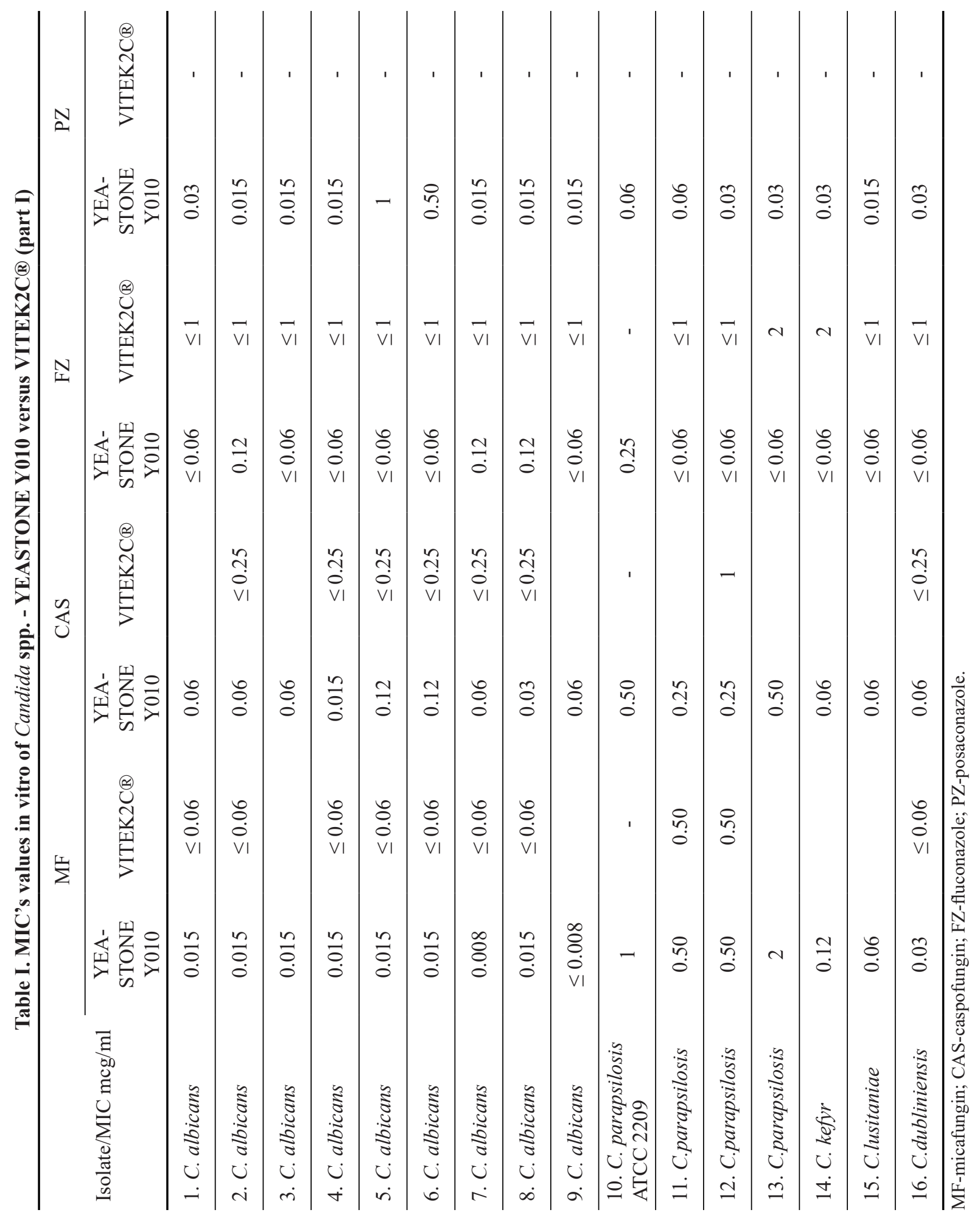




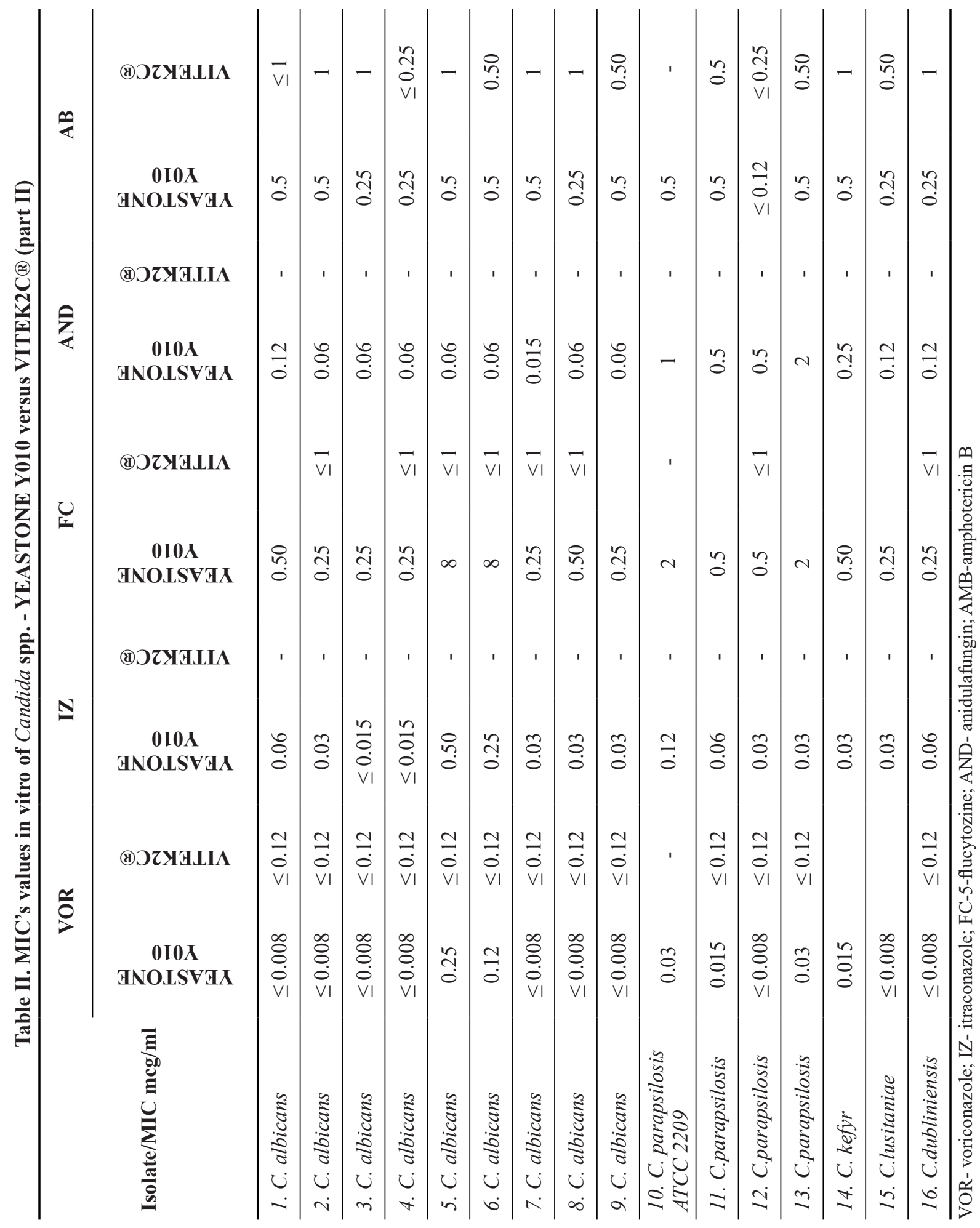


Table III. Categorically agreement (CA) for Candida spp. susceptibility to FLUCONAZOLE

\begin{tabular}{|c|c|c|c|c|c|c|c|c|c|c|c|c|}
\hline \multirow{2}{*}{$\begin{array}{l}\text { Fungus vs } \\
\text { Fluconazole } \\
\end{array}$} & \multicolumn{4}{|c|}{ YEASTONE® } & \multicolumn{4}{|c|}{ VITEK2C@ } & \multirow{2}{*}{ Correspondence } & \multicolumn{3}{|c|}{ Errors } \\
\hline & $\mathrm{T}$ & $\mathrm{S}$ & $\mathrm{I}$ & $\mathrm{R}$ & $\mathrm{T}$ & $\mathrm{S}$ & I & $\mathrm{R}$ & & Minor & Major & Very major \\
\hline C. albicans & 8 & 8 & 0 & 0 & 8 & 8 & 0 & 0 & $8(100 \%)$ & 0 & 0 & 0 \\
\hline C. parapsilosis & 3 & 3 & 0 & 0 & 3 & 3 & 0 & 0 & $3(100 \%)$ & 0 & 0 & 0 \\
\hline C. glabrata & 1 & 1 & 0 & 0 & 1 & 1 & 0 & 0 & $1(100 \%)$ & 0 & 0 & 0 \\
\hline C. kefyr & 1 & 1 & 0 & 0 & 1 & 1 & 0 & 0 & $1(100 \%)$ & 0 & 0 & 0 \\
\hline C. Iusitaniae & 1 & 1 & 0 & 0 & 1 & 1 & 0 & 0 & $1(100 \%)$ & 0 & 0 & 0 \\
\hline C. dubliniensis & 1 & 1 & 0 & 0 & 1 & 1 & 0 & 0 & $1(100 \%)$ & 0 & 0 & 0 \\
\hline Global & 15 & 15 & 0 & 0 & 15 & 15 & 0 & 0 & $15(100 \%)$ & 0 & 0 & 0 \\
\hline
\end{tabular}

$\mathrm{T}$ - total number of species; $\mathrm{S}$ - number of species with susceptibility ; I - number of species with intermediary; $\mathrm{R}$ - number of species with resistance to antifungals.

Table IV. Categorically agreement (CA) for Candida spp. susceptibility to AMPHOTERICIN B

\begin{tabular}{|c|c|c|c|c|c|c|c|c|c|c|c|c|}
\hline \multirow{2}{*}{$\begin{array}{l}\text { Fungus vs } \\
\text { Amphotericin B }\end{array}$} & \multicolumn{4}{|c|}{ YEASTONE® } & \multicolumn{4}{|c|}{ VITEK2C® } & \multirow{2}{*}{ Correspondence } & \multicolumn{3}{|c|}{ Errors } \\
\hline & $\mathrm{T}$ & $\mathrm{S}$ & I & $\mathrm{R}$ & $\mathrm{T}$ & $\mathrm{S}$ & I & $\mathrm{R}$ & & Minor & Major & Very major \\
\hline C. albicans & 8 & 8 & 0 & 0 & 8 & 8 & 0 & 0 & $8(100 \%)$ & 0 & 0 & 0 \\
\hline C. parapsilosis & 3 & 3 & 0 & 0 & 3 & 3 & 0 & 0 & $3(100 \%)$ & 0 & 0 & 0 \\
\hline C. glabrata & 1 & 1 & 0 & 0 & 1 & 1 & 0 & 0 & $1(100 \%)$ & 0 & 0 & 0 \\
\hline Global & 12 & 12 & 0 & 0 & 12 & 12 & 0 & 0 & $12(100 \%)$ & 0 & 0 & 0 \\
\hline
\end{tabular}

$\mathrm{T}$ - total number of species; $\mathrm{S}$ - number of species with susceptibility; I - number of species with intermediary susceptibility; $\mathrm{R}$ - number of species with resistance to antifungals.

For C. kefyr, C. lusitaniae, $\boldsymbol{C}$. dubliniensis it has been no data on EUCAST and CLSI.

Table V. Categorically agreement (CA) for Candida spp. susceptibility to VORICONAZOLE

\begin{tabular}{|c|c|c|c|c|c|c|c|c|c|c|c|c|}
\hline \multirow{2}{*}{$\begin{array}{l}\text { Fungus vs } \\
\text { Voriconazole }\end{array}$} & \multicolumn{4}{|c|}{ YEASTONE® } & \multicolumn{4}{|c|}{ VITEK2C® } & \multirow{2}{*}{ Correspondence } & \multicolumn{3}{|c|}{ Errors } \\
\hline & $\mathrm{T}$ & $\mathrm{S}$ & I & $\mathrm{R}$ & $\mathrm{T}$ & $\mathrm{S}$ & I & $\mathrm{R}$ & & Minor & Major & Very major \\
\hline C. albicans & 8 & 8 & 0 & 0 & 8 & 8 & 0 & 0 & $8(100 \%)$ & 0 & 0 & 0 \\
\hline C. parapsilosis & 3 & 3 & 0 & 0 & 3 & 3 & 0 & 0 & $3(100 \%)$ & 0 & 0 & 0 \\
\hline C. glabrata & 1 & 1 & 0 & 0 & 1 & 1 & 0 & 0 & $1(100 \%)$ & 0 & 0 & 0 \\
\hline Global & 12 & 12 & 0 & 0 & 12 & 12 & 0 & 0 & $12(100 \%)$ & 0 & 0 & 0 \\
\hline
\end{tabular}

$\mathrm{T}$ - total number of species; $\mathrm{S}$ - number of species with susceptibility; I - number of species with intermediary susceptibility; $\mathrm{R}$ - number of species with resistance to antifungals.

For C.kefyr, C.lusitaniae,C. dubliniensis - no data on EUCAST and CLSI were available to compare.

voriconazole, echinocandins (caspofungin and anidulafungin) were de-escaladed based on the susceptibility to antifungals generated by VITEK2C $®$ in $37 \%$ of cases.

\section{Discussions}

Regarding identifications of isolates and the study importance, being a retrospective study, one of the limitations revolves around the low 
number of isolates tested on micro broth dilutions (only 15) in comparison with 79 obtained from sterile sites on Vitek $2 C \AA$, namely that some did not cultivate after passing form bank to Sabouraud agar. Another limitation is that some species of Candida were not identified on VITEK $2 \mathrm{C} \AA(5.45 \%)$. VITEK system can cause errors - for example 4 cases ( 8 isolates from blood cultures) of C.auris, first cases identified in Europe, a species alerted via CDC alerting system, resistant to fluconazole and voriconazole, with susceptibility conserved to posaconazole, itraconazole, echinocandines și amphotericin B identified using ITS rDNA was considered by VITEK technology as C.lusitaniae, C.haemulonii while 6 isolates remained unidentified [10]. Using ITS "internal transcribed spacer" of ribosomal DNA some species identified on VITEK can be reconsidered and those unidentified can be precisely identified as Merseguel et al. identified 300 species in IFI [11]. It is possible that some isolates be misidentified as literature presents.

For susceptibility data, some studies identified differences between MIC's versus Clinical and Laboratory Standards (CLSI) breakpoints established in $2012-96.5 \%$ in C. albicans, $85.8 \%$ in C. tropicalis and $92.1 \%$ in C.parapsilosis according to the revised CBP's fluconazole susceptibility [12]. Sensititre YEASTONE compared with CLSI standards modified in 2012 regarding susceptibility to echinocandins generated only $1 \%$ errors [13]. Sensititre YEASTONE a microbroth dilution method used in clinical practice [14] obtained values of susceptibility in percentages of $98.7 \%$ in C. albicans, $92.5 \%$ in C.glabrata $92.3 \%$ in the C. parapsilosis complex, $96.1 \%$ in C. tropicalis and $100 \%$ in $C$. Guillermondii [15] for voriconazole and fluconazole this proving the accuracy of the method. Vijgen et al. identified in 2011 a concordance of $78.4 \%, 84.6 \%$ and $90.8 \%$ for fluconazole, voriconazole and amphotericin B between Vitek 2 and Sensititre YeastOne (SYO) [16]. Farina et al. in 2011, in a cooperation microbiology project which evaluated susceptibilities of 70 isolates of Candida on VITEK2 System and Sensititre YeastOne ${ }^{\circledR}$ to amphotericin B, voriconazol, fluconazol, flucytosine showed the results which credited VITEK system with a concordance for amphotericin B, fluconazole, voriconazole and 5-flucytozine (from $81.4 \%$ to $88.6 \%$ ). The researchers recommend VITEK with the mention to readjust the breakpoints [17].

In the present study, obtaining the MICs of Candida spp. was essential for epidemiological data and the errors between VITEK ${ }^{\circledR}$ MIC's and MIC's obtained by YEASTONE with 0 errors were crucial for clinicians' confidence and also for understanding the limits of the actual systems and potential of new technologies.

YEASTONE is correlated $100 \%$ with VITEK $2 \AA$ in terms of susceptibility. No antifungal resistance acquired events were reported in comparison with one study from Italy also using the YEASTONE panel where the fluconazole resistance on Candida spp. decreases to $5.4 \%$ in 2016 from $24.9 \%$ in a survey from 2009 [18]. Because no resistance to fluconazole was noticed in the susceptibility data we consider that first choice of fluconazole in medical practice the proper option when candidemia is suspected even if guidelines recommend echinocandins for candidemia $[2,19]$. Susceptibility to azoles, echinocandins, and amphotericin B in Romanian species involved in infectious diseases IFI is preserved in comparison with a 6 years' analysis in Switzerland (FUNGINOS project) where resistant isolates were mentioned [20].

\section{Conclusions}

The incidence in proven candidemia was 3.7 on 1,000 hospitalized patients. In these IFI patients, HIV infection was present in $27 \%$ of patients, predominantly males from urban areas with age limits between 12-83 years old. All our 
YEASTONE susceptibility determined data ( $\mathrm{S}$ -susceptible, I-intermediary, R-resistant) corresponded $100 \%$ to VITEK2 $\AA$ technology with no antifungal acquired resistance even on identification VITEK had some limitations. No resistance to any antifungal was noticed. Proportionally, the first option of treatment was fluconazole, followed by voriconazole and caspofungin, anidulafungin.

\section{Conflict of interest}

The authors declare that they have no conflict of interest.

\author{
Abbreviations \\ BSI- Bloodstream Infections \\ CDC-Centers for Disease Control and Preven- \\ tion \\ CLSI-Clinical and Laboratory Standards Insti- \\ tute \\ EUCAST-European Committee on Antimicrobi- \\ al Susceptibility Testing \\ FUNGINOS- Fungal Infection Network of Swit- \\ zerland \\ HAI-Hospital acquired infections \\ MIC-Minimum Inhibitory Concentration \\ ICU-Intensive Care Unit \\ IFI-Invasive Fungal Infections \\ ITS -Internal Transcribed Spacer \\ SDD-sensibility dose dependent \\ SYO-Sensititre YEASTONE
}

\section{References}

1. O'Neill J. Tackling drug-resistant infections globally: final report and recommendations, the review on antimicrobial resistance, Based on United Nations report World Population Prospects: The 2015 Revision, 2015, which cites current world population of 7.3 billion and projected world population in 2015 in 2050 of 9.7 billion. 2016;(5):12-3.
2. Pappas PG, Kauffman CA, Andes DR., Clancy CJ, Marr KA, Ostrosky-Zeichner L, et al. Clinical Practice Guideline for the Management of Candidiasis 2016. Clin Infect Dis. 2016; 62(4):e1-50. DOI: 10.1093/cid/ civ1194

3. Kullberg BJ, Arendrup M. Invasive candidiasis. N Engl J Med. 2015;373:1445-56. DOI: 10.1056/NEJMra1315399

4. Cornely OA, Gachot B, Akan H, Bassetti M, Uzun O, Kibbler C. et al. On behalf of the EORTC Infectious Diseases Group, Epidemiology and Outcome of Fungemia in a Cancer Cohort of the Infectious Diseases Group (IDG) of the European Organization for Research and Treatment of Cancer (EORTC 65031). Clin Infect Dis. 2015;61(3):324-31. DOI: 10.1093/cid/ civ293

5. Maschmeyer G,Patterson TF. Our 2014 aproach to breakthrough invasive fungal infections, Mycoses. 2014;57:645-51. DOI: 10.1111/myc.12213

6. Pfaller MA, Moet GJ, Messer SA, Jones RN, Castanheira M. Candida bloodstream infections: comparison of species distributions and antifungal resistance patterns in community-onset and nosocomial isolates in the SENTRY Antimicrobial Surveillance Program, 20082009. Diagn Microbiol Infect Dis. 2012;74(4):32331. DOI: 10.1016/j.diagmicrobio.2012.10.003

7. Minea B, Nastasa V, Moraru RF, Kolecka A, Flonta MM, Marincu I, et al. Species distribution and susceptibility profile to fluconazole, voriconazole and MXP4509 of 551 clinical yeast isolates from a Romanian multi-centre study. Eur J Clin Microbiol Infect Dis. 2015; 34(2): 367-83. DOI: 10.1007/s10096-014-22406

8. van Hal SJ, Chen SC, Sorrell TC, Ellis DH, Slavin M, Marriott DM. Support for the EUCAST and revised CLSI fluconazole clinical breakpoints by Sensititre ${ }^{\circledR}$ YeastOne ${ }^{\circledR}$ for Candida albicans: a prospective observational cohort study. J Antimicrob Chemother. 2014;69(8):2210-14. DOI: 10.1093/jac/dku124

9. *** 029-YEAST - ROW-IVD CID8962. www.thermoscientific.com/contactus. Accessed at 10.09.2017.

10. Merseguel KB, Nishikaku AS, Rodrigues AM, Padovan AC, Ferreira RC, de Azevedo Melo AS, et al.Genetic diversity of medically important and emerging Candida species causing invasive infection. BMC Infect Dis. 2015; 15:57. DOI: 10.1186/s12879-015-0793-3

11. Ruiz Gaitán AC, Moret A, López Hontangas JL, Moli- 
na JM, Aleixandre López AI, Cabezas AH, et al. Nosocomial fungemia by Candida auris: First four reported cases în continental Europe. Rev Iberoam Micol. 2017;34(1):23-7. DOI: 10.1016/j.riam.2016.11.002

12. Chen YC, Kuo SF, Chen FJ, Lee CH. Antifungal susceptibility of Candida species isolated from patients with candidemia in southern Taiwan, 2007-2012: impact of new antifungal breakpoints. Mycoses. 2017;60(2):8995. DOI: $10.1111 /$ myc. 12553

13. Pfaller MA, Chaturvedic V, Diekema DJ, Ghannoum M, Hollidaye NM, Killiane SB, et al. Comparison of the Sensititre Yeast One colorimetric antifungal panel with CLSI microdilution for antifungal susceptibility testing of the echinocandins against Candida spp., using new clinical breakpoints and epidemiological cutoff values. Diagn Microbiol and Infect Dis. 2012;73:365-8. DOI: 10.1016/j.diagmicrobio.2012.05.008

14. Kuykendall RJ, Lockhart SR. Microbroth Dilution Susceptibility Testing of Candida species. Methods Mol Biol. 2016;1356:173-81. DOI: 10.1007/978-1-49393052-4 13

15. Posteraro B, Spanu T, Fiori B, DeMaio F, De Carolis E, Giaquinto A, et al. Antifungal Susceptibility Profiles of Bloodstream Yeast Isolates by Sensititre Yeast One ever Nine Years at a Large Italian Teaching Hospital. Antimicrob Agents Chemother. 2015; 59(7):3944-55. DOI: 10.1128/AAC.00285-15

16. Vijgen S, Nys S, Naesens R, Magerman K, Boel A, Cartuyvels R. Comparison of Vitek identifica- tion and antifungal susceptibility testing methods to DNA sequencing and Sensititre YeastOne antifungal testing. Med Mycol. 2011;49(1):107-10. DOI: 10.3109/13693786.2010.494255

17. Farina C, Manso E, Andreoni S, Conte M, Fazii P, Lombardi $\mathrm{G}$, et al.Interlaboratory evaluation of VITEK2 system and Sensititre YeastOne ${ }^{\circledR}$ for antifungal susceptibility testing of yeasts isolated from blood cultures against four antifungal agents. New Microbiol. 2011;34(2):195-201.

18. Prigitano A, Cavanna C, Passera M, Ossi C, Sala E, Lombardi G, et al. CAND-LO 2014-15 study: changing epidemiology of candidemia in Lombardy (Italy). Infection. 2016;44(6):765-80. DOI: 10.1007/s15010016-0951-6

19. Leroux S, Ullmann AJ. Management and diagnostic guidelines for fungal diseases in infectious diseases and clinical microbiology: critical appraisal. Clin Microbiol Infect. 2013;19:1115-21. DOI: 10.1111/14690691.12426

20. Orasch C, Marchetti O,Garbino J, Schrenzel J, Zimmerli S, Muhlethaler K, et al. Candida species distribution and antifungal susceptibility testing according to European Committee on Antimicrobial Susceptibility Testing and new vs old Clinical and Laboratory Standards Institute clinical breakpoints: a 6 year prospective candidaemia survey from the fungal infection network of Switzerland. Clin Microbiol Infect. 2014;20:698705. DOI: $10.1111 / 1469-0691.12440$ 